\title{
Radial motion bias in macaque frontal eye field
}

\author{
QUAN XIAO, ANDREI BARBORICA, AND VINCENT P. FERRERA \\ Columbia University, Department of Psychiatry, Center for Neurobiology and Behavior, \\ David Mahoney Center for Brain and Behavior Research, New York, New York
}

(Received August 30, 2005; AcCEPTED September 15, 2005)

\begin{abstract}
The visual responsiveness and spatial tuning of frontal eye field (FEF) neurons were determined using a delayed memory saccade task. Neurons with visual responses were then tested for direction selectivity using moving random dot patterns centered in the visual receptive field. The preferred axis of motion showed a significant tendency to be aligned with the receptive-field location so as to favor motion toward or away from the center of gaze. Centrifugal (outward) motion was preferred over centripetal motion. Motion-sensitive neurons in FEF thus appear to have a direction bias at the population level. This bias may facilitate the detection or discrimination of expanding optic flow patterns. The direction bias is similar to that seen in visual area MT and in posterior parietal cortex, from which FEF receives afferent projections. The outward motion bias may explain asymmetries in saccades made to moving targets. A representation of optic flow in FEF might be useful for planning eye movements during navigation.
\end{abstract}

Keywords: Monkey, Frontal eye field, Optic flow

\section{Introduction}

Optic flow is a potentially important cue for navigation as it carries information about the motion of the observer (Gibson, 1950; Longuet-Higgins \& Prazdny, 1980; Koenderink, 1986). As an observer moves through the environment, the pattern of motion imaged on the retina has a strong radial component (expansion/ contraction) that can be analyzed to determine the direction in which the observer is heading (Perrone, 1992; Perrone \& Stone, 1994; Lappe \& Rauschecker, 1994; Duffy, 2000; Britten \& Van Wezel, 2002; Warren et al., 2001). There are at least two mechanisms by which optic flow patterns might be represented in the brain. The flow might be represented explicitly by the firing of individual neurons that are specialized for nonlinear motion patterns, such as neurons in dorsal middle superior temporal area (MST) (Saito et al., 1986; Graziano et al., 1994), area 7a (Siegel \& Read, 1997; Merchant et al., 2003), ventral intraparietal (VIP) (Schaafsma \& Duysens, 1996), STPa (Anderson \& Siegel, 1999), and PEc (Raffi et al., 2002). Another form of optic flow sensitivity might be implicit in a population of linear motion detectors if the preferred direction of motion for each detector is correlated with its receptive-field position relative to the fovea such that the neuronal population shows a preference for radial motion. The latter type of organization has been reported in posterior parietal cortex (Steinmetz et al., 1987) and visual area MT (Albright,

Address correspondence and reprint requests to: Vincent P. Ferrera, Center for Neurobiology and Behavior, Columbia University, 1051 Riverside Drive, Kolb Annex 504, New York, NY 10032, USA. E-mail: vpf3@ columbia.edu
1989). The current study reports evidence for such a radial flow representation in the frontal eye field (FEF).

The frontal eye field is a region of prefrontal cortex that has reciprocal connections with extrastriate cortex, including parietal motion areas (Barbas \& Mesulam, 1981; Stanton et al., 1995; Schall et al., 1995). FEF is divided into subregions specialized for saccades (FEFs) and smooth pursuit (FEFsp) eye movements. Neurons in the smooth pursuit subregion are directionally selective for visual stimuli and eye movements (MacAvoy et al., 1991; Gottlieb et al., 1993, 1994). However, little is known about visual motion sensing properties of neurons in the saccade subregion of FEF. FEFs neurons respond selectively when saccade targets are chosen based on the direction of a moving dot field (Kim \& Shadlen, 1999). They are also selective for speed of target motion and this selectivity is sustained even if the target is rendered temporarily invisible (Barborica \& Ferrera, 2003).

In the present study, the saccade region of the FEF was located on the basis of eye movements evoked by electrical microstimulation. Cells in FEFs were tested for visual responsiveness using a memory-saccade task. Cells with visual responses (both visual and visual-movement cells) were then stimulated with moving random dot patterns placed within the receptive field. Neuronal activity was analyzed to determine the robustness of direction and speed tuning and the preferred direction of motion relative to receptivefield location. Most FEFs neurons showed significant direction tuning and the preferred direction tended strongly to be aligned with outward radial motion. This form of representational bias has not been described in FEF and suggests that FEF may play a larger role in optic flow analysis and visual navigation than has previously been suggested. A radial motion bias is certainly not incom- 
patible with a more explicit representation of optic flow. The present results may warrant a more thorough exploration of FEF neuronal responses to genuine optic flow patterns.

\section{Materials and methods}

Experiments were performed on five subadult male rhesus monkeys (Macaca mulatta). The treatment of the monkeys was in accordance with the guidelines set by the US Department of Health and Human Services (NIH) for the care and use of laboratory animals, and all methods were approved by the Institutional Animal Care and Use Committee at Columbia University and the New York State Psychiatric Institute. Monkeys were prepared for experiments by surgical implantation of a post for head restraint and a recording chamber to give access to the cortex. Eye position was recorded using a monocular scleral search coil (Judge et al., 1980). All surgical procedures were performed using aseptic technique and general anesthesia (isoflurane 1-3\%). Monkeys were trained to sit in a primate chair for the duration of each experiment with their heads restrained. Correct performance was reinforced by liquid reward.

\section{Visual stimulation}

Visual stimuli were generated and controlled by a Cambridge Research Systems VSG2/3F video frame buffer. The output from the video board was displayed on a calibrated 29 or 37 inch color monitor (Mitsubishi, Irvine, CA) with a $60-\mathrm{Hz}$ noninterlaced refresh rate. The monitor stood at a viewing distance of 24 or 30 inch (depending on monitor size) so that the display area subtended roughly $40 \mathrm{deg}$ horizontally by $30 \mathrm{deg}$ vertically. The spatial resolution of the display was 1280 pixels by 1024 lines. Fixation targets were small $(0.5 \mathrm{deg})$ white squares presented on a uniform black background. The luminance of the fixation target was $65.0 \mathrm{~cd} / \mathrm{m}^{2}$, while the background was close to $0 \mathrm{~cd} / \mathrm{m}^{2}$ (below the photometer threshold). Moving random dot stimuli were generated by placing $2 \times 2$ pixel $(0.06 \times 0.06 \mathrm{deg})$ white dots at random positions within a circular aperture and displacing each dot by a fixed increment on every frame. The dots all moved in the same direction at the same speed (100\% coherence) and the lifetime of the dots was equal to the length of the stimulus presentation $(500 \mathrm{~ms})$. The dots were randomly redrawn before each trial so that any given pattern of dots was unlikely to be repeated. Dot density was $0.5 / \mathrm{deg}^{2}$. The frame buffer was programmed to send out digital pulses (frame sync) for timing purposes at the beginning of each video frame in which a target was turned on or off. These pulses were recorded by the computer using a hardware timer (Lisberger Technologies, San Francisco, CA), and stored together with the neuronal and eye movement data.

\section{Neuronal recording and electrical stimulation}

A recording chamber (20-mm diameter) was implanted on the skull overlying the arcuate sulcus. The recording chamber was positioned at stereotaxic coordinates 25A, 15L (Paxinos et al., 2000). At the start of each recording session, a hydraulic microdrive was mounted on the recording chamber. Recordings were made using platinum-iridium or tungsten electrodes with impedances of $0.3-2 \mathrm{M} \Omega @ 1 \mathrm{kHz}$. Signals from the microelectrode were amplified, filtered, and monitored on an oscilloscope and audio monitor. A time-amplitude window discriminator converted extracellular action potentials into digital pulses (TTL) which were sampled by the computer with $0.01-\mathrm{ms}$ time resolution. Units were isolated on the basis of waveform. When a unit was isolated, stimulus parameters such as position and size were adjusted to optimize its response. Neuronal spike trains were collected and stored along with eye position and velocity records.

Electrical microstimulation was used to map the region of cortex from which neuronal recordings were obtained in each monkey. Sites in peri-arcuate cortex were stimulated through the same electrode used to record neuronal activity. The stimulation consisted of a train of $0.2-\mathrm{ms}$ biphasic pulses at a rate of 350 pulses/s delivered by an optically isolated pulse stimulator (AM Systems, Seattle, WA). The output of the stimulator was gated by a computer-generated TTL level so as to be synchronized with other trial events. The current threshold for evoking saccades was determined by stimulating during a fixation task (Opris et al., 2001). The threshold was defined as the current level at which involuntary saccades were evoked on about half the stimulation trials (Bruce et al., 1985). For 284 peri-arcuate sites the median threshold was $42.5 \mu \mathrm{A}(\min 10 \mu \mathrm{A}, \max 100 \mu \mathrm{A})$.

The arcuate sulcus could be visualized transdurally during the recording chamber implantation surgery. The position of the sulcus was confirmed by making long electrode penetrations (up to $10 \mathrm{~mm}$ below the cortical surface) during which action potentials characteristic of neuronal cell bodies could be continuously recorded as the electrode advanced, indicating that the tip of the electrode remained in gray matter throughout the penetration. Fig. 1A shows a coronal magnetic resonance image (MRI) for one monkey $(\mathrm{F})$ with an electrode track $(*)$ clearly visible in the anterior bank of the arcuate sulcus. Fig. 1B shows saccades evoked during a fixation task by suprathreshold electrical stimulation at the site marked by the asterisk. For all sites, electrically evoked saccades were almost always contraversive and showed a mediolateral gradation of amplitudes (Bruce \& Goldberg, 1985). In addition, the evoked saccade direction rotated systematically as the depth of the electrode changed. These features of the saccade amplitude and direction map are characteristic of the FEF. The median electrically evoked saccade amplitude for 284 stimulation sites was $4.05 \mathrm{deg}$ ( $\min 0.4 \mathrm{deg}$, max $18.7 \mathrm{deg}$, interquartile range $3.0-6.95 \mathrm{deg})$. Only $36 / 284(13.6 \%)$ of the sites had characteristic saccades larger than $10 \mathrm{deg}$, indicating that nearly all of the recording and stimulation sites were in the region of the FEF that represents small-to-medium amplitude saccades. Smooth pursuit eye movements were evoked at a few stimulation sites, but none of the recordings for this study were made at those sites.

\section{Behavioral paradigms}

Monkeys were trained to perform two oculomotor tasks during neuronal recording; a memory-saccade task (MEM), and a fixation task with passive presentation of motion stimuli (DOTS). Horizontal and vertical eye position were sampled at $1 \mathrm{kHz}$ per channel and digitized with 12-bit resolution. In the MEM task, monkeys made saccades to the remembered location of a visual cue. The cue location varied among eight positions, equally spaced (45 deg) around the clock (Fig. 2A; Funahashi et al., 1989). At the beginning of each trial the monkey fixated a small white square (Fig. 2B). A peripheral cue was flashed for $750 \mathrm{~ms}$ followed by a variable delay (750-1250 ms) during which the fixation target remained on and the monkey maintained fixation within a $2 \times 2 \mathrm{deg}$ window. At the end of the delay, the fixation target disappeared and the monkey was allowed up to $800 \mathrm{~ms}$ to make a saccade to the remembered location of the cue. After the 800-ms saccade interval, and if the monkey's memory saccade was within a $3 \times 3 \mathrm{deg}$ 

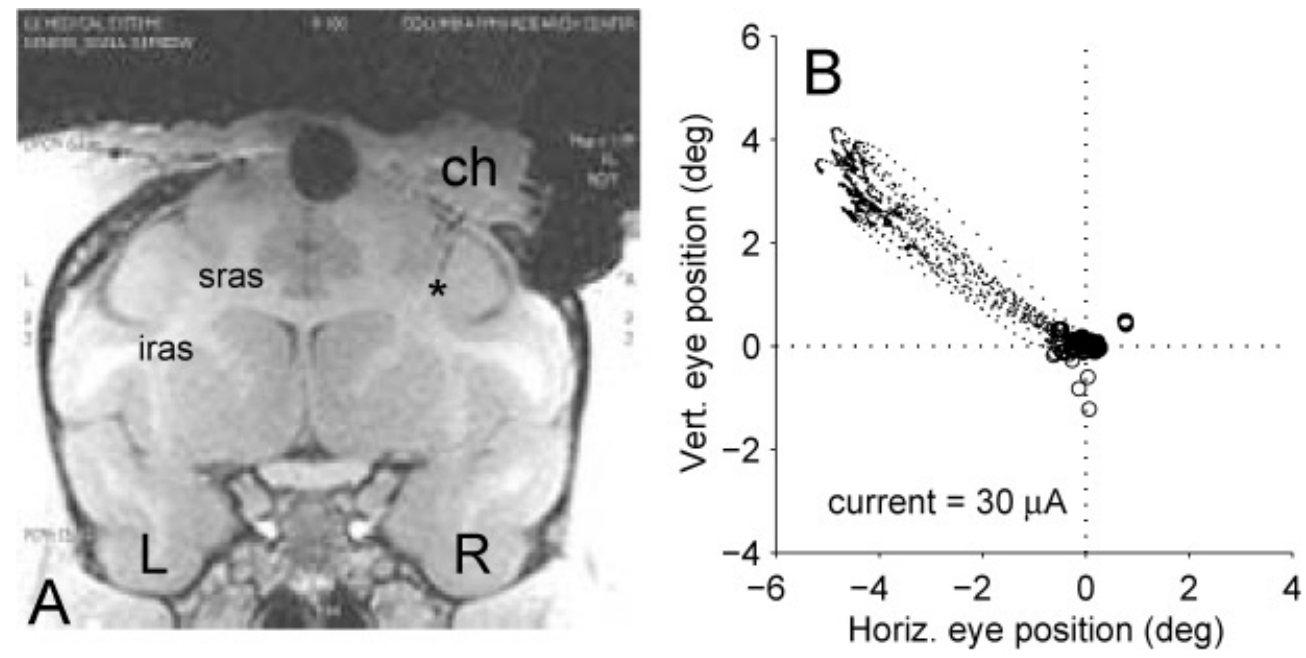

Fig. 1. Verification of recording/stimulation sites. (A) Coronal MRI at the level of the arcuate sulcus; left and right hemispheres are labeled. "iras" and "sras" are the inferior and superior rami of the arcuate sulcus, respectively. "ch" is the recording chamber in the right hemisphere. Asterisk indicates electrode track. (B) Contraversive saccades evoked by suprathreshold stimulation at the site in (A) indicated by “*” (threshold was $25 \mu \mathrm{A}$ ).

window centered on the cue location, the cue reappeared to provide feedback to the monkey and corrective saccades were generally made at this time. The eccentricity of the peripheral cue was varied to find the optimum eccentricity for each neuron before data were recorded. Data were then recorded with this fixed eccentricity.

In the DOTS task, the monkey maintained fixation within a $2 \times$ 2 deg window centered around the fixation target while the random moving dot stimuli were presented in the periphery (Fig. 2B). The stimulus duration was $500 \mathrm{~ms}$. On each trial, a random direction and speed were chosen from a set of eight directions (0-315 deg, 45 -deg increments) and three speeds $(5,10, \& 20 \mathrm{deg} / \mathrm{s})$. The reason for not using faster speeds was to avoid aliasing artifacts due to the refresh rate of the monitor. The size of the dot aperture was generally $8 \mathrm{deg}$, but could be varied to optimize the response of each cell.
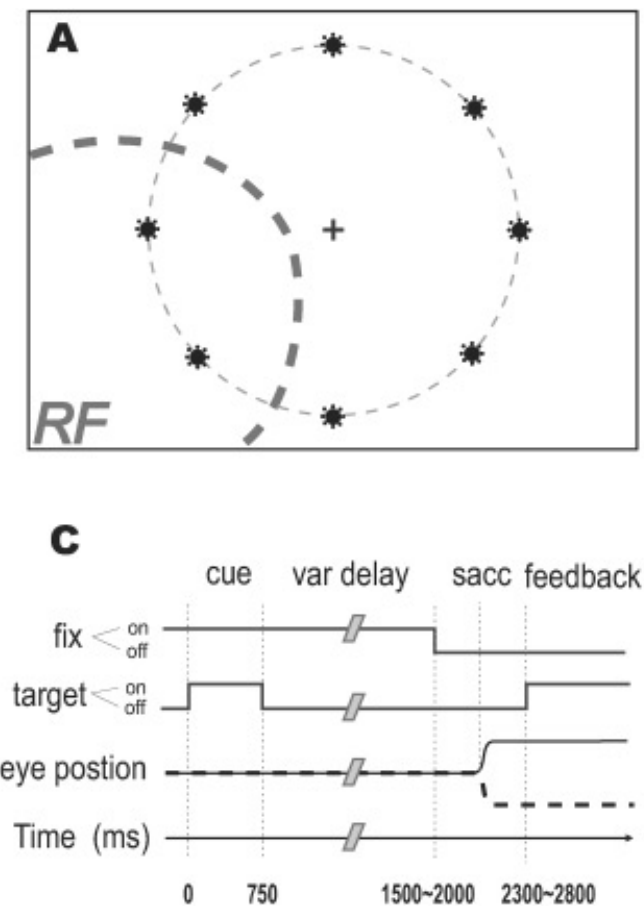

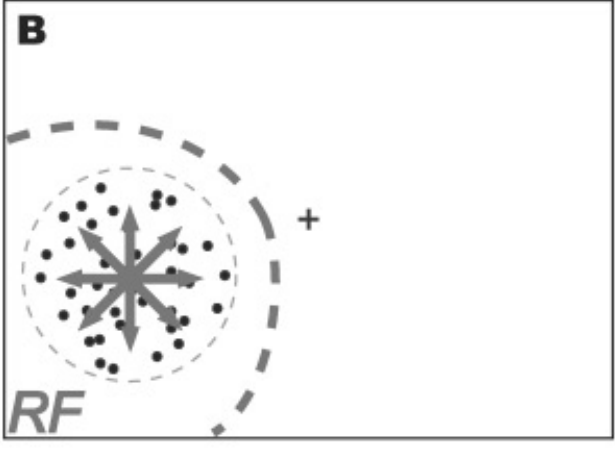

D

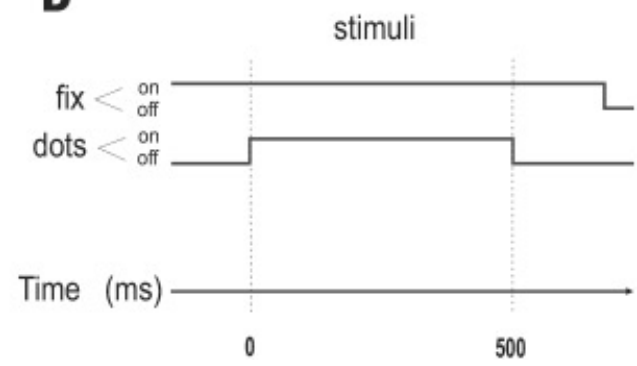

Fig. 2. Behavioral paradigms. (A) Memory-saccade task (MEM). (B) Fixation task with passive presentation of random moving dot patterns (DOTS). Event timing for MEM task. (C) Event timing for DOTS task. Event timing for MEM task. (D) Event timing for DOTS task. 
After all the neurons for this study had been recorded, monkeys were trained to perform a modified version of the DOTS task. In the modified task, the central fixation target was extinguished and monkeys were rewarded for making a saccade that landed within $\pm 5 \mathrm{deg}$ of the center of the dot pattern (10-deg diameter). Monkeys were given feedback regarding the accuracy of their saccade by placing a small $(0.25 \mathrm{deg})$ white stationary target at the center of the dot pattern. The feedback target appeared $600 \mathrm{~ms}$ after the onset of the dot pattern. The saccade was always initiated before the appearance of the feedback target. The dot pattern was placed at 10 -deg eccentricity. The position, direction, and speed of the dot pattern were chosen randomly for each trial from a set of eight positions (equally spaced at 45-deg intervals on the perimeter of an imaginary 10-deg radius circle), eight directions (4 cardinals plus 4 obliques), and two speeds (stationary and $20 \mathrm{deg} / \mathrm{s}$; total $=72$ trials). Each trial type was repeated 28 times for a total of 2000 trials per monkey.

\section{Statistics}

Data were analyzed with standard tests such as ANOVA and Rayleigh's test for circular uniformity (Zar, 1999). To these we added an iterative resampling procedure to compare the circular dispersion of two angle distributions. On each iteration, half the data were selected at random from each distribution. Each subsample was used to construct a resultant vector by treating each observed angle, $\theta$, as a unit vector pointing in the direction of $\theta$. The unit vectors were summed and normalized by the number of observations to yield the resultant. The length of the resultant vector, $|R|$, varies between 0 and 1 , and is an index of the concentration of the original angle distribution. The procedure was iterated 20,000 times and the two distributions of resultant vector magnitudes were compared using an unpaired $t$-test.

\section{Results}

We recorded the activity of 199 neurons in the frontal eye field (FEFs) of five monkeys (monkey A: $n=33$; C: $n=65$; D: $n=29$;
$\mathrm{E}: n=20 ; \& \mathrm{~F}: n=52)$. In the majority of cases, each neuron was first tested with the MEM task to determine the relative strength of the visual and presaccadic responses, and the preferred location for visual stimuli. Cells with visual responses were then tested with the DOTS task with the stimulus centered at the preferred location. A total of 154/199 (77\%) neurons satisfied the condition that at least five blocks of data were recorded for both the MEM and DOTS tasks (monkey A: $n=16$; C: $n=52$; D: $n=24$; E: $n=14$; F: $n=46$ ). For the remaining 45 neurons, data were collected only for the DOTS task, at least five blocks, and the receptive field was located by moving the dot stimulus around the screen until the best response was obtained. The center of the dot stimulus was taken to be the preferred receptive-field location.

The activity of a single FEF neuron is shown in Fig. 3. In Fig. 3A spike times recorded during the MEM task were aligned to the onset of the visual cue. The dark regions of the peristimulus time histograms highlight the visual response. The spatial tuning of the visual response (average firing rate during cue interval) is shown in the central plot. The arrow indicates the center-of-mass $(C M)$ vector computed from the tuning curve as

$$
C M=\sum\left(\mathbf{u}_{i} * f_{i}\right) / \sum\left(f_{i}\right),
$$

where $\mathbf{u}_{i}$ is a unit vector pointing to the $i$ th target and $f_{i}$ is the average firing rate associated with that target direction.

The target eccentricity used for the MEM task for this unit was 10 deg. However, this cell responded quite well over a broad range of eccentricities, as was typical for neurons in our sample. For the DOTS task, the stimulus was placed $7.5 \mathrm{deg}$ to the left of fixation and $7.5 \mathrm{deg}$ below the horizontal meridian. Activity as a function of dot speed and direction is shown in Fig. 3B. The central plot shows tuning curves and center-of-mass vectors for each speed. This cell was strongly directional but responded equally well to all three speeds (two-way ANOVA, factor $=$ direction, $P<0.0001$; factor $=$ speed, $P=0.24$ ). For all three speeds, the preferred direction, indicated by the $C M$ vectors, was within 60 deg of the preferred location vector determined by the MEM task. The peaks of the direction and spatial responses were both at $225 \mathrm{deg}$.

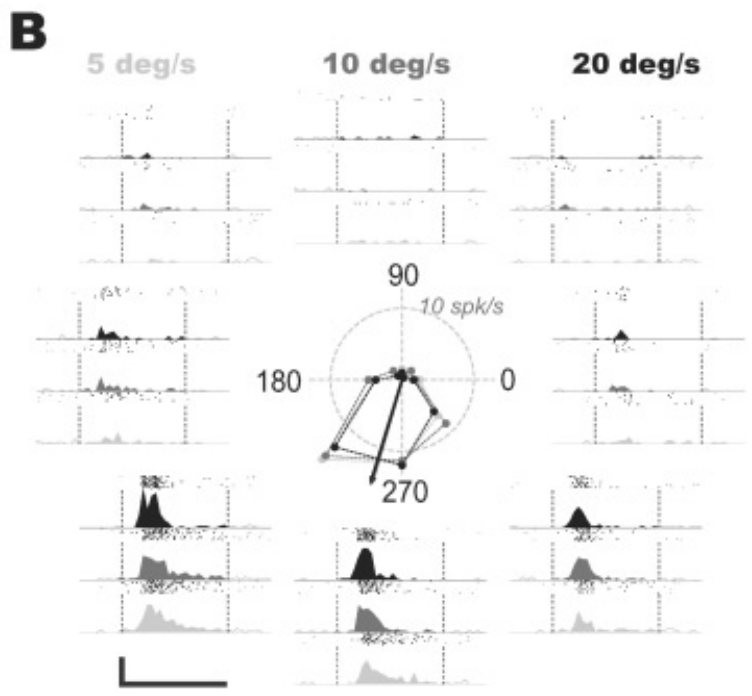

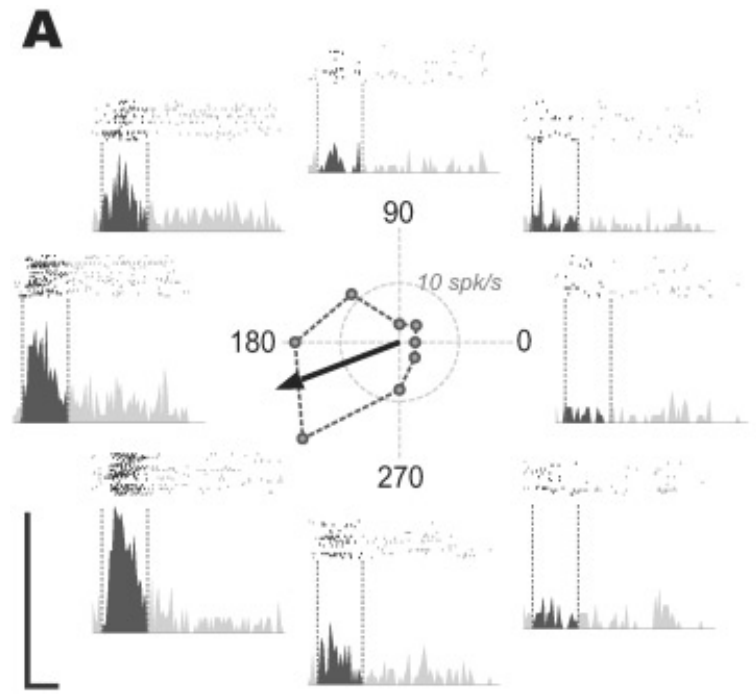

Fig. 3. Single neuron response during MEM and DOTS tasks. (A) Activity as a function of target location for the MEM task. Each subplot contains a raster where each dot indicates the occurrence of a spike and each line corresponds to a single trial. Below each raster is a peristimulus time histogram (PSTH) where black indicates the ON response to the visual cue and gray indicates activity outside the cue interval. The central plot shows the average cue response as a function of cue location and the large arrow is the center-of-mass (CM) vector. (B) Activity during the DOTS task sorted by stimulus direction and speed. Same conventions as (A). 


\section{Direction and speed selectivity}

Selectivity for motion parameters was assessed using a two-way ANOVA (factors: motion direction and speed). The unit shown in Fig. 3B had a highly significant effect of direction $(P<0.001)$, but not speed $(P=0.24)$. For the population, 104/199 $(52 \%)$ of the neurons showed a significant effect of direction $(P<0.05)$. For speed, 80/199 (40\%) had a significant effect. These numbers indicate that a substantial proportion of cells were selective for each stimulus dimension but should not be directly compared as it is unlikely that the range of stimulus speeds was well matched to the full range of speeds over which FEF neurons might respond.

Direction selectivity was quantified using a direction index $(D I)$ defined as

$$
D I=(P-N) /(P+N),
$$

where $P$ is the average firing rate evoked by a stimulus moving in the preferred direction and $N$ is the average firing rate in the null (180 deg opposite preferred) direction. The median direction index was $0.24(n=199)$, which corresponds to a preferred-null ratio of 1.63:1.

\section{Radial motion bias}

The relative preferred direction of each neuron was determined by calculating its preferred direction ( $P D$ vector) of motion during the DOTS task, and the vector connecting the fixation point to the preferred location ( $R F$ vector) of the neuron (which was identical to the location of the dot pattern). The relative preferred direction is the angular difference between the directions of the $P D$ and $R F$ vectors. Fig. 4A shows the angular distribution of relative preferred directions for all 199 neurons. Zero degrees corresponds to a preferred direction moving outward from the fixation point, while $180 \mathrm{deg}$ corresponds to inward motion. Ninety and $270 \mathrm{deg}$ correspond to clockwise and counterclockwise rotation. The circular mean $(\theta)$ of the distribution of relative preferred directions was $23.7 \mathrm{deg}$ and a Rayleigh test (Zar, 1999) found the probability that the distribution was circularly uniform to be $P<0.001$. Note that in Fig. 4A, as in some subsequent figures, the main histogram peak is not always centered at 0 deg. This is partly a sampling issue and partly a binning issue. The data were sampled at 45-deg intervals, both in position and direction, hence one expects some unevenness in the angle distributions. Furthermore, histogram binning can make the distributions appear less uniform than they really are. When the data are resampled in various ways, the most consistent feature is a peak near $0 \mathrm{deg}$, while other peaks tend to appear less consistently.

If the radial bias represents a specialization for motion processing, then one might expect the strength of the bias to be correlated with direction selectivity (DS). To test this, the direction index [eqn. (2)] was used to divide the cells into two classes: strongly direction selective (DI greater than the median $D I$ for all cells) and weakly DS ( $D I \leq$ median). Figs. $4 \mathrm{~B}$ and $4 \mathrm{C}$ show the distributions of relative preferred directions for these two classes of cell. Both distributions show a strong and statistically significant radial outward bias (Rayleigh test; $P<0.001$ ). However, the distribution of strongly DS cells has more cells concentrated near the $0-180$ axis than the distribution for weakly DS cells. As a result of this, the dispersion for the distribution of strongly DS cells $(|R|=0.25)$ was more uniform than the dispersion of the weakly DS cells $(|R|=0.32)$. An iterative resampling procedure (see Methods, Statistics) found that the resultant magnitude for the weakly DS cells was larger than that for the strongly DS cells on $76 \%$ of the iterations. The difference in resultant magnitudes was highly significant (unpaired $t$-test, $P<0.0001$ ).

\section{Movement planning}

It is could be argued that the radial motion bias is related to saccade planning. If monkeys covertly planned a saccade to the motion stimulus and if some aspect of this plan were correlated with motion direction, then this covert plan might introduce an
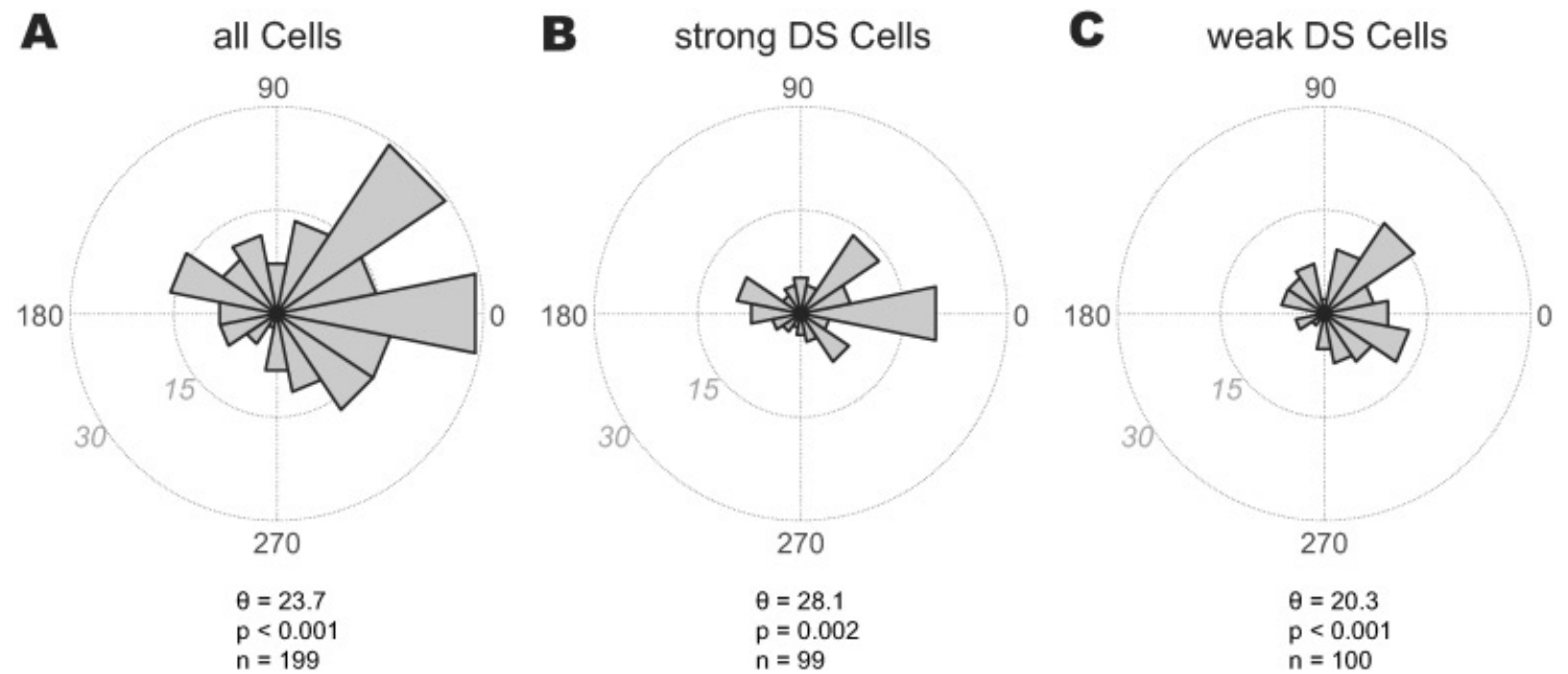

Fig. 4. Distributions of relative preferred directions. (A) Angle histogram for all cells. Zero degrees corresponds to radial outward motion preference. $\theta$ is the circular mean of the distribution; $P$ is the significance level for the Rayleigh test; and $n$ is the number neurons. (B) Angle histogram for the most direction selective (DS) neurons. (C) Angle histogram for the least DS neurons. Same conventions as (A). 
apparent bias in the direction preferences of the cells. To address this, we separated the cells into "visual" and "movement" categories based on activity during the MEM task. The visual ( $V$, average firing rate during cue period) and presaccadic ( $S$, average firing rate $0-100 \mathrm{~ms}$ preceding memory saccade) activities of each neuron were used to calculate a visual-movement index as follows:

$$
V M I=(V-S) /(V+S)
$$

This index ranges from 1.0 (pure visual cell) to -1.0 (pure movement cell). The VMI distribution for 154 neurons tested with the MEM task was approximately Gaussian with a median of -0.015 . The median $V M I$ was used to split the population into two equal halves. Cells with VMIs larger than the median were classified as "visual," and cells with VMIs less than the median were classified as "movement."

Assuming that movement plans are more strongly represented by presaccadic activity than by visually driven activity, the movement planning hypothesis predicts that the radial motion bias should be stronger for movement cells than for visual cells. The relative preferred direction distributions are shown for "visual" cells in Fig. 5A and for "movement" cells in Fig. 5B. The visual cells appeared to show a stronger outward bias than the movement cells. This was confirmed by computing the dispersion for visual $(|R|=0.34)$ and movement $(|R|=0.26)$ cells. The distribution for visual cells was significantly more concentrated (unpaired $t$-test on resampled data, $P<0.0001)$.

If the stimulus direction-dependent aspect of the motor plan begins with the onset of the stimulus and builds up over time, the movement planning hypothesis also predicts that the preferred direction of the neuron should evolve over the timecourse of the stimulus presentation. To explore this, the neuronal population response was computed by averaging the responses from all neurons (Fig. 6A). Neuronal activity for each trial was divided into nonoverlapping 20-ms time bins. The average number of spikes was computed for each bin, the average background activity $(-200$ to $0 \mathrm{~ms}$ relative to stimulus onset) was subtracted, and the result was averaged over all neurons. Fig. 6A shows the average response to preferred (thick line) and antipreferred (thin line) directions. In both cases, the population response started at around $40-60 \mathrm{~ms}$ and peaked between 80 and $100 \mathrm{~ms}$ after stimulus onset. (In anesthetized macaques, visual latencies in FEF can be as short as 50-60 ms, Schmolesky et al. 1998.) The population response begins to show direction selectivity within $60 \mathrm{~ms}$ of stimulus onset (Fig. 6A, dashed line).

To determine the time dependence of the radial direction bias, the 500-ms stimulus interval was divided into ten nonoverlapping 50-ms subintervals. The direction tuning of each neuron was computed within each subinterval. The bias in the distribution of relative preferred direction vectors was estimated by computing the resultant vector. The bias was strongest and most significant (Rayleigh test) between 150 and $200 \mathrm{~ms}$ after stimulus onset (Fig. 6B), when the resultant vector magnitude reached a value $>0.2$. Yet, there was a significant bias as early as $50-100 \mathrm{~ms}$ after stimulus onset, which corresponds to the earliest directionselective population response. The bias continued to evolve for the next 100-150 ms before starting to decline.

Samples of the relative preferred direction distribution for two time intervals are shown in Figs. 6C and 6D. The direction tuning for each neuron was calculated using only the initial (first 50$200 \mathrm{~ms}$ ) or only the terminal $(350-500 \mathrm{~ms})$ part of the stimulus presentation. The relative preferred direction distribution for the initial response (Fig. 6C) was significantly more concentrated than the distribution based on the later part of the stimulus interval
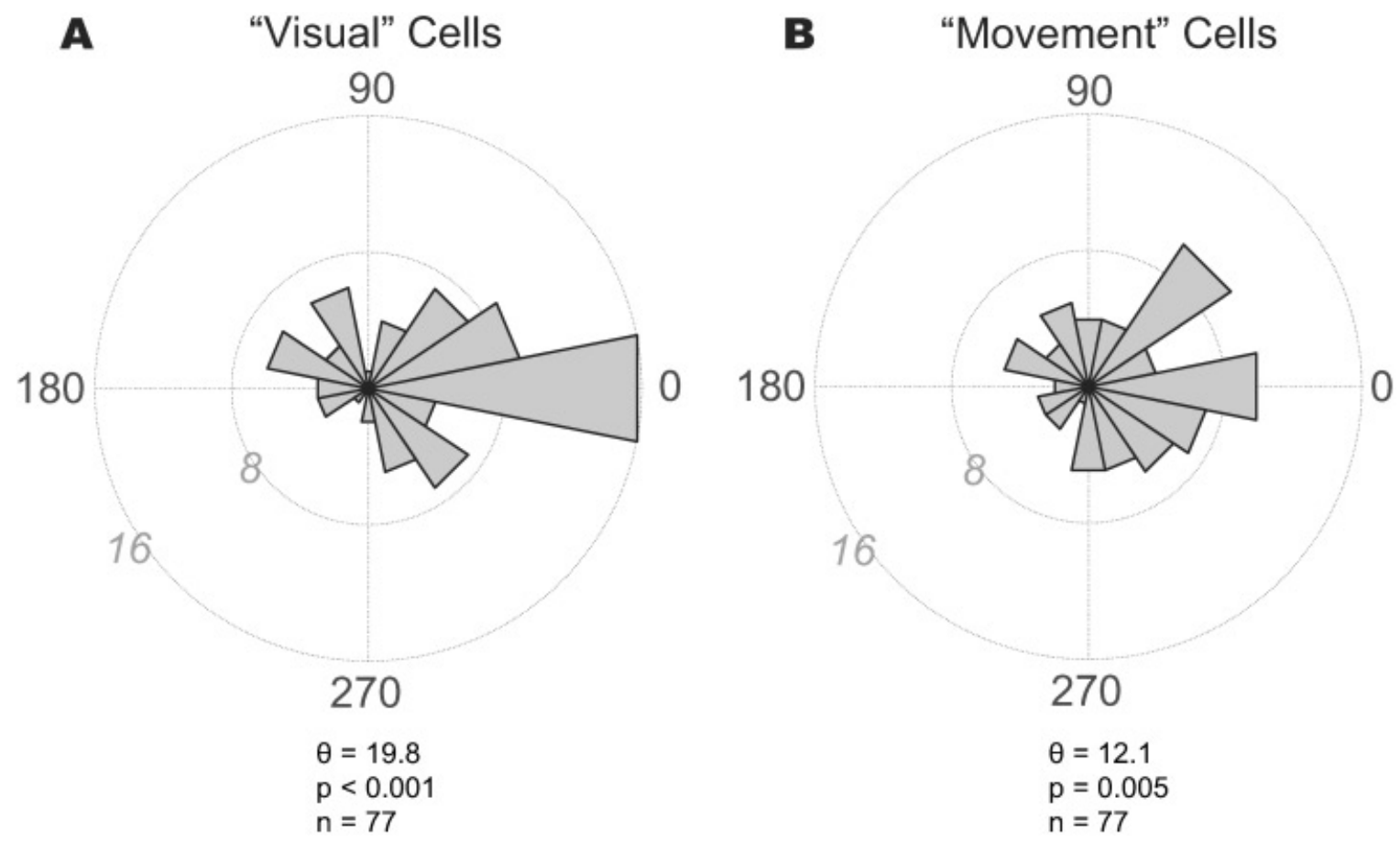

Fig. 5. Angle histograms for relative preferred directions of visual (A) and movement (B) cells as classified by activity during memory-guided saccades. Same conventions as in Fig. 4. 

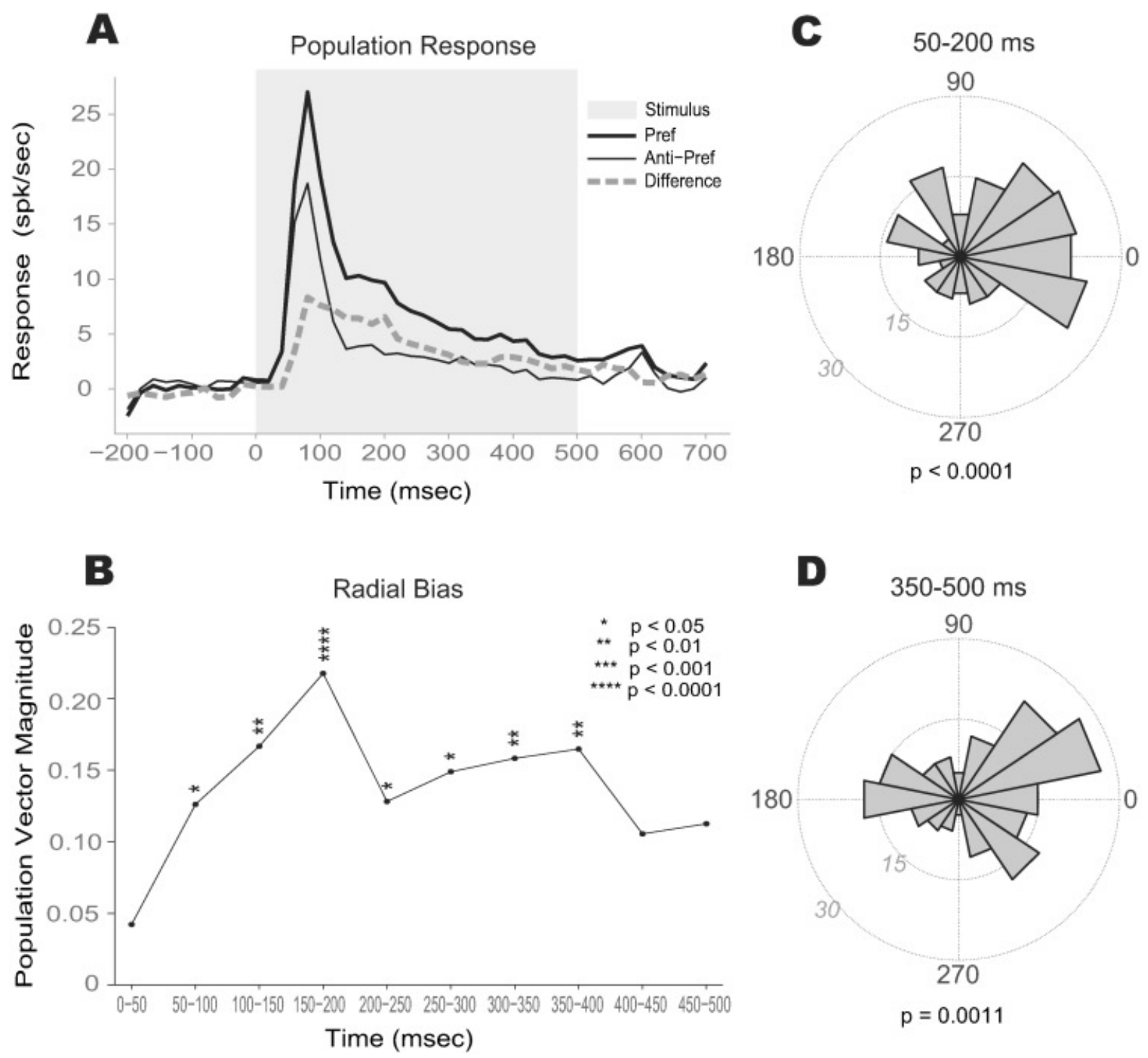

Fig. 6. Neuronal population response to moving dot patterns. (A) Average firing rate for preferred direction (thick black line), anti-preferred direction (thin black line), and preferred-antipreferred difference (dashed line). (B) Vector magnitude of direction bias as a function of time during stimulus presentation. Asterisks indicate significance level of Rayleigh test. (C) Angle histograms for relative preferred direction based on initial part of the stimulus interval, or (D) end of the stimulus interval. Same conventions as in Fig. 4.

(Fig. 6D). The length of the resultant for the initial response was 0.29 compared to 0.18 for the later response $(P<0.0001$; unpaired $t$-test on resampled data).

\section{Eye movements during fixation}

Attention and/or movement plans are potentially affected by stimulus motion. To find evidence of this, we analyzed eye movements that were recorded at the same time as the neuronal data. These eye movement records included both microsaccades and slow drifts. Fig. 7A shows radial eye velocity (Pythagorean sum of horizontal and vertical velocities) averaged over all trials, neurons, and monkeys. The initial decay that occurred between 0 and $200 \mathrm{~ms}$ before stimulus onset is due to the tail end of the saccade made to the fixation target at the beginning of each trial. There then an initial dip followed by a rise in eye velocity that began $100 \mathrm{~ms}$ into the trial and peaked at $2.6 \mathrm{deg} / \mathrm{s}$ just after the stimulus had been on for 200-300 ms. This peak was due to an increased probability of microsaccades and slow drifts. This peak was seen in all five monkeys, although the exact timing varied (monkey A: 149, C: 275, D: 200, E: 196, \& F: 248 ms). The peak was therefore contemporaneous with the peak of the radial bias, but was well after the initial direction selective visual response and also after the time when a significant radial bias first was detected in the neuronal data.

To analyze fixational eye movements in more detail, we calculated the direction of the eye movement in relation to the radial component of stimulus motion (i.e., the direction of stimulus velocity relative to the direction of stimulus position). Fig. 7B shows the eye velocity amplitude (averaged over all monkeys) as 

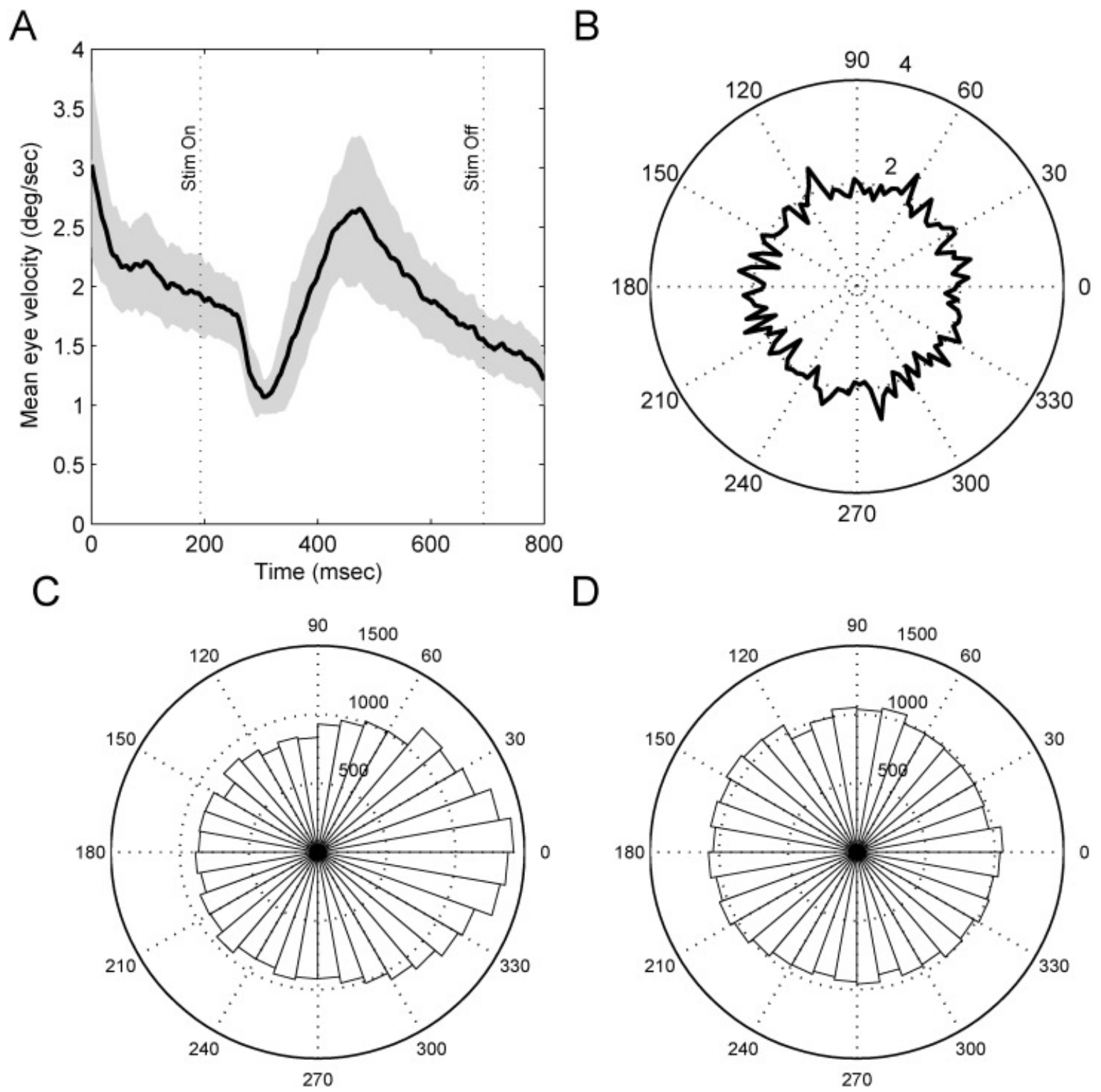

Fig. 7. Analysis of fixational eye movements during neurons recording. (A) Radial eye velocity averaged over all trials and monkeys. Gray area indicates \pm 1 S.D. (B) Amplitude of radial eye velocity as a function of relative stimulus direction (direction of stimulus motion-direction of stimulus position). (C) Direction of eye velocity relative to absolute stimulus motion direction. (D) Direction of eye velocity with respect to relative stimulus direction.

a function of relative stimulus direction. For each monkey, eye velocity on each trial was extracted by averaging over a 50 -ms window centered on the time of the peak eye velocity for that monkey. The effect of stimulus motion was not significant (oneway ANOVA, $P=0.8$ ). Hence the average amplitude of the fixational movements did not vary with stimulus motion.

Fig. 7C shows an angle histogram of the direction of the eye movements relative to absolute stimulus direction. There were significantly more eye movements aligned with target motion than with other directions (Rayleigh test, $P<0.00001$ ). However, to show that there was a radial bias, it is necessary to plot eye movement direction relative to the radial component of stimulus motion (direction of stimulus motion-direction of stimulus position). The distribution of these angles is plotted in Fig. 7D. The distribution appears uniform, although the Rayleigh test was highly significant $(P<0.00001$; most likely this significance level was due to the slight elongation of the distribution along the horizontal axis coupled with the large number of observations). However, the critical comparison is between relative directions of 0 and $180 \mathrm{deg}$. The differences in the numbers of eye movements around these directions are negligible.

\section{Saccades to motion stimuli}

The time at which the radial bias peaked (150-200 ms) is in the range of visually guided saccade latencies in monkeys, leaving open the possibility that the bias is related to saccade planning. If this were the case, then one might expect to see similar biases in the latency or amplitude of voluntary saccades made to the moving dot stimuli. We therefore trained all five monkeys to perform a 
modified version of the DOTS task in which they made saccades from the fixation point to the center of the moving dots. The patterns were centered at $10-\mathrm{deg}$ eccentricity. This training was undertaken after all neuronal data were collected. We measured the direction, amplitude, accuracy, and latency of each saccade. Data were plotted as a function of the relative direction of the position and velocity vectors for each stimulus (Fig. 8;0 deg $=$ outward motion, $180 \mathrm{deg}=$ inward motion).

Fig. 8A shows saccade direction relative to the center of the stimulus. Saccades to stationary stimuli had a systematic error of $2.3 \mathrm{deg}$. (This systematic error in saccade direction is actually quite small. It means that, for a 10-deg saccade, the endpoint of the saccade typically missed the center of the stimulus by $0.4 \mathrm{deg}$ of visual angle.) For moving stimuli, saccade direction was influenced by stimulus motion (one-way ANOVA, $P<0.05$ ). However, the effect was driven by directions of motion orthogonal to saccade direction (90 and $270 \mathrm{deg})$. For radial motion ( 0 vs. $180 \mathrm{deg})$, there was no effect on saccade direction.
Fig. 8B shows normalized saccade amplitude as a function of relative direction of motion. Saccade amplitude was normalized to the median amplitude for each monkey and then combined for all monkeys. Saccades to outward moving patterns were $10 \%$ longer on average (median amplitude) than those to inward moving patterns. The effect of relative direction was significant $(P<$ 0.0001; one-way ANOVA). The post-hoc comparison between saccade amplitudes for 0 and $180 \mathrm{deg}$ was also significant $(P<$ 0.001 ; unpaired $t$-test). The dashed lines indicate median normalized saccade amplitude \pm 1 S.D. for saccades to zero-velocity patterns at the same retinal eccentricity $(10 \mathrm{deg})$. Thus, saccades to moving dot patterns had amplitudes comparable to those made to stationary patterns. The effect of motion was to bias saccade amplitude slightly in the direction of motion.

All saccades had a tendency to undershoot the center of the pattern (median non-normalized amplitude range: 9.1-10.4 deg; average $=9.3 \pm 1.4$ S.D. deg). Thus, saccades to outward moving patterns were more accurate by $20 \%$ than those to inward moving
A Direction

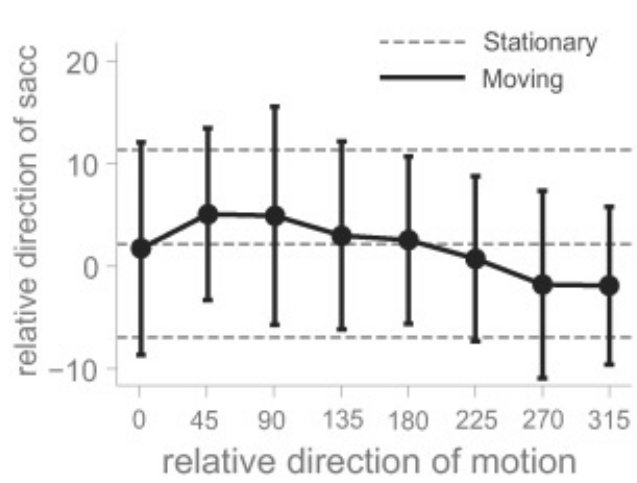

relative direction of motion

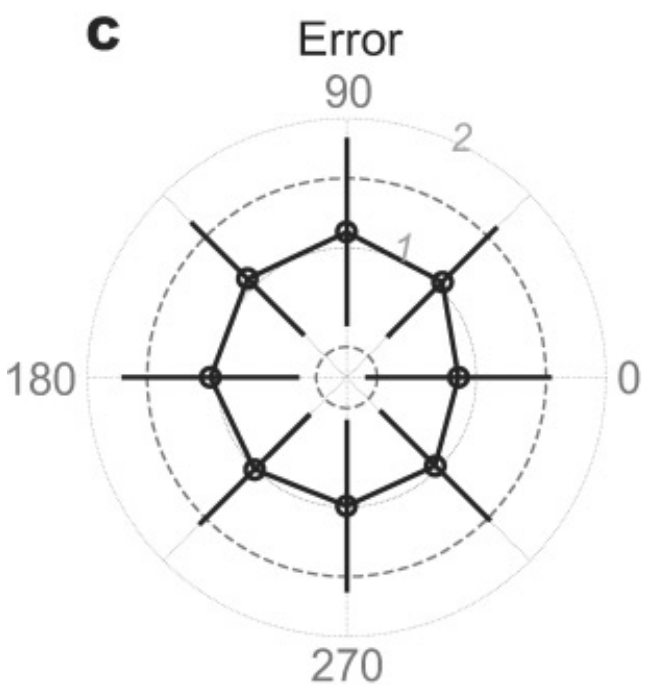

B Amplitude
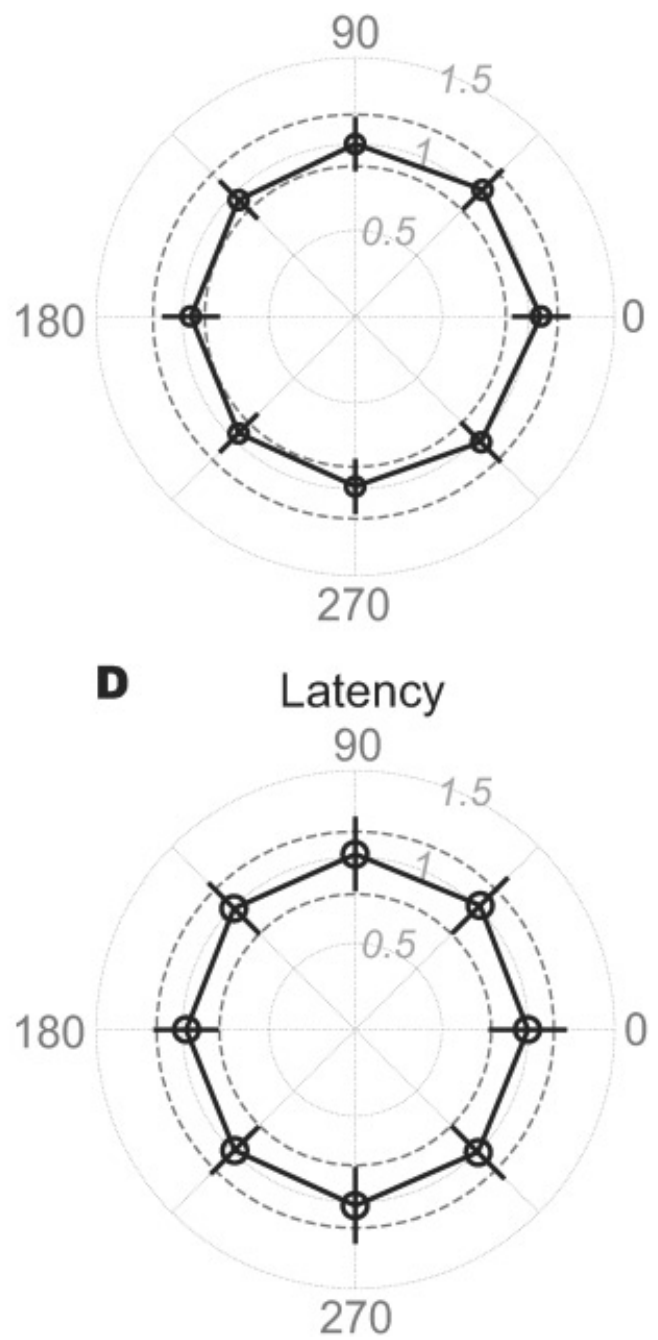

Fig. 8. Parameters of saccades to moving and stationary patterns. (A) Saccade direction (median \pm 1 S.D.) relative to center of stimulus as a function of relative direction of motion. Dashed lines are median saccade direction ( \pm 1 S.D.) for stationary stimuli. (B) Filled circles represent normalized amplitude (median \pm 1 S.D.) of saccades to moving patterns. Dashed lines indicate median \pm 1 S.D. amplitude of saccades to stationary patterns. (C) Normalized saccade error. (D) Normalized saccade latency. 
targets. Fig. 8C shows the normalized saccade error. The effect of relative direction was significant $(P>0.0001$; one-way ANOVA; post-hoc t-test $0 v s .180 \operatorname{deg} P<0.001)$. Dashed lines indicate the range of saccade errors (median \pm 1 S.D.) for stationary patterns. Saccade latency showed no dependence on relative pattern motion (Fig. 8D). Median saccade latency varied across animals in the range of 163 to $221 \mathrm{~ms}$ with S.D.'s ranging from $30 \mathrm{~ms}$ to $47 \mathrm{~ms}$.

\section{Discussion}

The population of FEF neurons recorded in this study showed a highly significant bias for preferring outward motion when tested with moving random dot patterns. A significant radial bias was present in the earliest direction-selective population response. The bias reached a peak 150-200 ms after stimulus onset. Saccadic eye movements directed toward motion stimuli also showed a small but significant outward bias. These results suggest that projections to FEF from posterior visual areas preserve the radial motion bias seen in MT and posterior parietal cortex (Steinmetz et al., 1987; Albright, 1989), and that this bias is incorporated into motor commands for saccades. A radial motion bias has also been reported for cells in the lateral suprasylvian cortex of cats (LS; Rauschecker et al., 1987), and recent studies have found evidence for an explicit representation of optic flow in cat LS ( $\mathrm{Li}$ et al., 2000; Sherk \& Fowler, 2001). The preferred orientation of neurons in V1 also shows a radial bias (Bauer \& Dow, 1989; Bauer et al., 1983; Leventhal, 1983; Vidyasagar \& Henry, 1990), suggesting that this form of representational bias may be ubiquitous in visual cortex.

A more explicit form of optic flow representation has been well documented in posterior parietal cortex, starting with the work of Saito et al. (1986) in area MST. It is not clear whether area MT plays a direct role in the encoding of optic flow, nor is it known whether the radial bias for preferred direction in MT contributes to the optic flow sensitivity of neurons in MST and elsewhere. The finding that FEFs has a similar bias to that seen in MT does not firmly establish a role in optic flow processing and visual navigation for either structure, but it does hint that such a role may be possible.

Human functional imaging studies that have used optic flow stimuli have not reported significant activation in frontal eye field (Wunderlich et al., 2002; Greenlee, 2000; Morrone et al., 2000; Dukelow et al., 2001; Peuskens et al., 2001). This might be due to low signal strength combined with high statistical thresholds for identifying significant blood oxygen level dependent signal (BOLD) activation. Using imaging paradigms and behavioral tasks that enhance signal strength may reveal motion and/or optic-flow specific responses in human FEF. To date, the vast majority of imaging and single-unit studies of optic flow have focused on parietal cortex. However, selective responses to optic flow stimuli have been found in motor cortex of monkeys (Merchant et al., 2001). The present results suggest that it should be worthwhile to look more closely at the role of prefrontal cortex in optic flow analysis and visual navigation.

\section{Perception of optic flow patterns}

The perceived speed of optic flow varies depending on the type of flow pattern. For humans, outward radial flow generally appears faster than inward flow, and both appear faster than rotational flow (Clifford et al., 1999). This would be consistent with the outward bias seen in MT and FEFs if one assumes that perceived speed is determined by a rate code where the rate is proportional to the aggregate firing of a population of neurons (i.e., the number of neurons responding and their rate of firing). This assumption may be warranted at least for speeds that are on the rising limb of the population speed tuning function (Priebe et al., 2003; Priebe \& Lisberger, 2004).

Perception of optic flow patterns degraded by noise also depends on the pattern of flow. Detection thresholds are lowest for radial (expansion/contraction) and rotational patterns, and are higher for spiral flow patterns that are intermediate between radial and rotational motion (Morrone et al., 1999). For discriminating direction of motion, thresholds for radial flow are slightly lower than those for concentric flow. This asymmetry could be supported by the radial bias seen in FEFs as well as other structures.

\section{Effects of visual motion on saccadic eye movements}

FEFs is involved in the planning and initiation of voluntary eye movements. One might therefore expect that a radial motion bias in FEFs would have consequences for saccades to moving targets. For example, saccades to small targets moving away from the fovea tend to be larger and have shorter latency than saccades to targets moving toward the fovea (Segraves et al., 1987). This asymmetry may be a consequence of the stronger population response to outward motion in FEF.

Small moving targets have both a net displacement as well as a direction of motion, whereas the windowed motion stimuli in the present experiment had no net displacement. These two stimulus types might have very different effects on eye movements. To determine the effects on saccadic eye movements of the motion stimuli used in this study, we trained monkeys to make saccades to stationary and moving dot patterns. Monkeys tended to undershoot the center of the target regardless of whether the dots were moving or stationary. When the dots were moving, saccades were both longer and more accurate for outward motion than for inward motion. The effect of motion on saccade amplitude resulted in a $10 \%$ difference between saccades to outward ( $0 \mathrm{deg})$ and inward (180 deg) moving stimuli. However, there was no effect of motion on saccade latency.

Analysis of fixational eye movements suggests that the motion stimulus did affect attention and/or movement planning. There was a significant effect of stimulus motion on fixational eye movements. However, this effect appeared after the time at which the radial bias in the neuronal data was already established. The amplitude and direction of fixational movements showed no bias with respect to the radial component of stimulus motion. For these reasons, it is extremely unlikely that attention and/or movement planning were the cause of the neuronal bias.

Horwitz and Newsome (2001) recorded from the superior colliculus in monkeys trained to use motion as a cue for saccade target selection. They reported a strong bias in preferred direction with most neurons preferring motion toward the direction of their movement fields. In those experiments, the motion stimulus was presented at the center of gaze, so that target location and, hence, saccade direction were perfectly correlated with stimulus direction. To rule out an effect of saccade planning, they performed a control experiment in which saccade direction was decoupled from stimulus motion direction and found that the preferred direction of the neurons remained aligned with the movement field. In our experiments, the stimulus position and direction of motion were never correlated and the monkeys were not trained to use stimulus motion as a cue for saccade selection. If monkeys always planned saccades toward the exact center of the stimulus, then there would 
be no correlation between saccade planning and stimulus motion because the saccades would have fixed amplitude and direction while the stimulus motion varied.

However, when monkeys were trained to make saccades to the motion stimulus, those saccades did not always land in the center of the stimulus, but were systematically biased by stimulus motion. This behavioral bias thus provides some evidence for stimulus motion effects on motor planning, although the effect was only a small deviation in the endpoint of saccades that were generally directed to the same location. In contrast, the design of Horwitz and Newsome's (2001) experiment was such that saccade direction could vary by $180 \mathrm{deg}$ depending on stimulus motion. In the current study, both the direction and amplitude of the saccades were affected. The effect on saccade direction showed no radial bias, but did show a significant effect of motion orthogonal to saccade direction. Thus, stimulus-induced differences in the planned direction of movement would be expected to produce a neuronal response bias for concentric (clockwise and counterclockwise) motion, not for radial motion.

The effect of stimulus motion on saccade amplitude might explain the radial bias in the neurons' preferred directions if neurons responded more when monkeys were planning larger saccades. Most FEFs neurons are broadly tuned for saccade amplitude, so a difference of $10 \%$ (1.0 deg of visual angle) would likely give rise to a small difference in neuronal response, and hence a weak direction bias. The movement planning hypothesis thus predicts that the most weakly direction-selective neurons should show the strongest radial bias. In fact, the data show that there was a significant radial bias for the most strongly direction selective neurons, whose directionality is least likely to be explained by small direction-dependent differences in saccade amplitude.

The time dependence of the neuronal bias suggests an alternative to the visual-movement dichotomy (i.e., the idea that the bias must be due either to afferent visual input or movement planning). As shown in Fig. 6, a significant radial bias is present in the earliest direction selective response in FEF. However, the bias continues to evolve over time. The bias reaches its peak at a time appropriate for the latency of saccades directed the stimulus. Thus, an alternative view is that the bias is partly due to afferent input, but is further elaborated over time and incorporated into the movement command that drives the eyes. If this view is correct, then the bias in saccade amplitude may be a consequence of the neuronal bias rather than the cause of it.

\section{Eye movements during navigation}

The integration of optic flow information in FEF could play a useful role in planning eye movements during navigational tasks such as driving (Land \& Lee, 1994; Wilkie \& Wann, 2003). Radial flow may highlight salient regions of the flowfield such as the focus of expansion, or scene elements such as curves that can be used to anticipate future changes in heading direction. Observers tend to direct their gaze preferentially toward such regions (Lappe et al., 1998). However, gaze direction during driving depends on the task conditions; when driving on straightaways, gaze tends to be directed toward the focus of expansion, but when negotiating curves, gaze tends to be directed toward the inner edge of the roadway (Lappe \& Hoffman 2000). These context-dependent differences in gaze strategy involve continuous oculomotor decision making and are therefore likely to engage FEFs (see Schall, 2002).

Navigation requires the coordination of eye movements in three-dimensional space. Radial optic flow has been found to induce vergence eye movements in humans (Busettini et al., 1997) and monkeys (Inoue et al., 1998). Centrifugal motion increases vergence angle thereby causing the plane of fixation to recede, an appropriate response for an observer moving forward. Recent evidence suggests a role for the FEF in controlling eye movements in three dimensions. Neurons in the smooth pursuit region of FEF (FEFsp) respond during vergence tracking (Fukushima et al., 2002). Neurons in the saccade region of FEF are sensitive to retinal disparity, which drives vergence (Ferraina et al., 2000). Microstimulation in the anterior bank of the arcuate sulcus can cause disjunctive eye movements (Gamlin \& Yoon, 2000). Although we were careful to avoid recording from FEFsp, it is possible that some of our recordings were located in the vergence area. FEFs might contribute to visual navigation by integrating optic flow and three-dimensional eye movements.

\section{Conclusions}

These experiments have demonstrated a strong bias for preferred motion direction in macaque FEFs. This representational bias may underlie behavioral biases in voluntary saccades to moving targets or windowed motion stimuli. These experiments suggest a previously unsuspected role for FEFs in the analysis of optic flow. The present observations do not rule out a more explicit representation of optic flow in FEFs. On the contrary, they suggest that it will be profitable search for such an explicit representation and to explore its integration with eye movement planning during tasks such as visual navigation or heading discrimination.

\section{Acknowledgments}

This research was supported by the Whitehall and James S.McDonnell Foundations and NIH MH59244.

\section{References}

Albright, T.D. (1989). Centrifugal directional bias in the middle temporal visual area (MT) of the macaque. Visual Neuroscience 2(2), 177-188.

Anderson, K.C. \& Siegel, R.M. (1999). Optic flow selectivity in the anterior superior temporal polysensory area, STPa, of the behaving monkey. Journal of Neuroscience 19(7), 2681-2692.

Barbas, H. \& Mesulam, M.M. (1981). Organization of afferent input to subdivisions of area 8 in the rhesus monkey. Journal of Comparative Neurology 200(3), 407-431.

BarboricA, A. \& Ferrera, V.P. (2003). Estimating invisible target speed from neuronal activity in monkey frontal eye field. Nature Neuroscience 6(1), 66-74.

BAuer, R. \& Dow, B.M. (1989). Complementary global maps for orientation coding in upper and lower layers of the monkey foveal striate cortex. Experimental Brain Research 76, 503-509.

Bauer, R., Dow, B.M., Synder, A.Z. \& Vautin, R.G. (1983). Orientation shift between upper and lower layers in monkey visual cortex. Experimental Brain Research 50, 133-145.

Britten, C.M. \& VAN Wezel, R.J. (2002). Area MST and heading perception in macaque monkeys. Cerebral Cortex 12(7), 692-701.

Bruce, C.J. \& GoldberG, M.E. (1985). Primate frontal eye fields. I. Single neurons discharging before saccades. Journal of Neurophysiology 53, $603-635$.

Bruce, C.J., Goldberg, M.E., Bushnell, M.C. \& Stanton, G.B. (1985). Primate frontal eye fields. II. Physiological and anatomical correlates of electrically evoked eye movements. Journal of Neurophysiology $\mathbf{5 3}$, $714-734$.

Busettini, C., Masson, G.S. \& Miles, F.A. (1997). Radial optic flow induces vergence eye movements with ultra-short latencies. Nature 390, 512-515.

Clifford, C.W.G., Beardsley, S.A. \& Vaina, L.M. (1999). The perception and discrimination of speed in complex motion. Vision Research 39, 2213-2227. 
DuFFy, C.J. (2000). Optic flow analysis for self-movement perception. International Review of Neurobiology 44, 199-218.

Dukelow, S.P., DeSouza, J.F., Culham, J.C., van den Berg, A.V., Menon, R.S. \& Vilis, T. (2001). Distinguishing subregions of the human MT+ complex using visual fields and pursuit eye movements. Journal of Neurophysiology 86(4), 1991-2000.

Ferraina, S., Pare, M. \& Wurtz, R.H. (2000). Disparity sensitivity of frontal eye field neurons. Journal of Neurophysiology 83(1), 625-629.

Fukushima, K., Yamanobe, T., Shinmei, Y., Fukushima, J., Kurkin, S. \& Peterson, B.W. (2002). Coding of smooth eye movements in three-dimensional space by frontal cortex. Nature 419, 157-162.

Funahashi, S., Bruce, C.J. \& Goldman-Rakic, P.S. (1989). Mnemonic coding of visual space in the monkey's dorsolateral prefrontal cortex. Journal of Neurophysiology 61, 331-349.

Gamlin, P.D. \& Yoon, K. (2000). An area for vergence eye movement in primate frontal cortex. Nature 407, 1003-1007.

Gibson, J.J. (1950). The Perception of the Visual World. Boston, Massachusetts: Hougton Mifflin.

Gottlieb, J.P., Bruce, C.J. \& MacAvoy, M.G. (1993). Smooth eye movements elicited by microstimulation in the primate frontal eye field. Journal of Neurophysiology 69(3), 786-799.

Gottlieb, J.P., MacAvoy, M.G. \& BRuce, C.J. (1994). Neural responses related to smooth-pursuit eye movements and their correspondence with electrically elicited smooth eye movements in the primate frontal eye field. Journal of Neurophysiology 72(4), 1634-1653.

Graziano, M.S., Andersen, R.A. \& Snowden, R.J. (1994). Tuning of MST neurons to spiral motions. Journal of Neuroscience 14(1), 54-67.

Greenlee, M.W. (2000). Human cortical areas underlying the perception of optic flow: brain imaging studies. International Review of Neurobiology 44, 269-292.

Horwitz, G.D. \& Newsome, W.T. (2001). Target selection for saccadic eye movements: Direction-selective visual responses in the superior colliculus. Journal of Neurophysiology 86, 2527-2542.

Inoue, Y., Takemura, A., Suehiro, K., Kodaka, Y. \& Kawano, K. (1998). Short-latency vergence eye movements elicited by looming step in monkeys. Neuroscience Research 32(2), 185-188.

JUdGE, S.J., RICHMOND, B.J. \& CHU, F.C. (1980). Implantation of magnetic search coils for measurement of eye position: An improved method. Vision Research 20, 535-538.

Kim, J.N. \& Shadlen, M.N. (1999). Neural correlates of a decision in the dorsolateral prefrontal cortex of the macaque. Nature Neuroscience 2(2), 176-185.

KoENDERINK, J.J. (1986). Optic flow. Vision Research 26, 161-180.

LAND, M.F. \& LeE, D.N. (1994). Where we look when we steer. Nature 369(6483), 742-744

Lappe, M. \& Hoffmann, K.-P. (2000). Optic flow and eye movements. In Neuronal Processing of Optic Flow, ed. LAPPE, M., Academic Press. International Review of Neurobiology 44, 29-47.

Lappe, M., Pekel, M. \& Hoffmann, K.-P. (1998). Optokinetic eye movements elicited by radial optic flow in the macaque monkey. Journal of Neurophysiology 79, 1461-1480.

LapPe, M. \& Rauschecker, J.P. (1994). Heading detection from optic flow. Nature 369, 712-713.

LeVenthal, A.G. (1983). Relationship between preferred orientation and receptive field position of neurons in cat striate cortex. Journal of Comparative Neurology 220, 476-483.

Li, B., Li, B.W., Chen, Y., Wang, L.H. \& Diao, Y.C. (2000). Response properties of PMLS and PLLS neurons to simulated optic flow patterns. European Journal of Neuroscience 12(5), 1534-1544.

Longuet-Higgins, H.C. \& Prazdny, K. (1980). The interpretation of a moving retinal image. Proceedings of the Royal Society B (London) 208(1173), 385-397.

MacAvoy, M.G., Gottlieb, J.P. \& Bruce, C.J. (1991). Smooth-pursuit eye movement representation in the primate frontal eye field. Cerebral Cortex 1(1), 95-102.

Merchant, H., Battaglia-Mayer, A. \& Georgopoulos, A.P. (2001). Effects of optic flow in motor cortex and area 7a. Journal of Neurophysiology 86(4), 1937-1954.

Merchant, H., Battaglia-Mayer A. \& Georgopoulos, A.P. (2003). Functional organization of parietal neuronal responses to optic-flow stimuli. Journal of Neurophysiology 90(2), 675-682.

Morrone, M.C., Burr, D.C., DiPietro, S. \& Stefanelli, M.-A. (1999). Cardinal directions for visual optic flow. Current Biology 9, 763-766.

Morrone, M.C., Tosetti, M., Montanaro, D., Fiorentini, A., Cioni,
G. \& BURR, D.C. (2000). A cortical area that responds specifically to optic flow, revealed by fMRI. Nature Neuroscience 3(12), 1322-1328.

Opris, I., Barborica, A. \& Ferrera, V.P. (2001). On the gap effect for saccades evoked by electrical microstimulation of frontal eye fields in monkeys. Experimental Brain Research 138, 1-7.

Peuskens, H., Sunaert, S., Dupont, P., Van Hecke, P. \& Orban, G.A. (2001). Human brain regions involved in heading estimation. Journal of Neuroscience 21(7), 2451-2461.

Priebe, N.J. \& Lisberger, S.G. (2004). Estimating target speed from the population response in visual area MT. Journal of Neuroscience $\mathbf{2 4 ( 8 )}$ 1907-1916.

Priebe, N.J., Cassanello, C.R. \& Lisberger, S.G. (2003). The neural representation of speed in macaque area MT/V5. Journal of Neuroscience 23(13), 5650-5661.

Paxinos, G., Huang, X.F. \& Toga, A.W. (2000). The Rhesus Monkey Brain in Stereotaxic Coordinates. San Diego, CA: Academic Press.

Perrone, J.A. (1992). Model for the computation of self-motion in biological systems. Journal of the Optical Society of America A 9, 177-194.

Perrone, J.A. \& Stone, L.S. (1994). A model of self-motion estimation within primate extrastriate visual cortex. Vision Research 34, 2917-2938.

Raffi, M., SQuatrito, S. \& Maioli, M.G. (2002). Neuronal responses to optic flow in monkey parietal area PEc. Cerebral Cortex 12, 639-646.

Rauschecker, J.P., von Grunau, M.W. \& Poulin, C. (1987). Centrifugal organization of direction preferences in the cat's lateral suprasylvian visual cortex and its relation to optic flow field processing. Journal of Neuroscience 7, 943-958.

Saito, H., Yukie, M., Tanaka, K., Hikosaka, K., Fukada, Y. \& Iwai, E. (1986). Integration of direction signals of image motion in the superior temporal sulcus of the macaque monkey. Journal of Neuroscience $\mathbf{6 ( 1 )}$, $145-157$.

SchAAFsma, S.J. \& DuYSEns, J. (1996). Neurons in the ventral intraparietal area of awake macaque monkey closely resemble neurons in the dorsal part of the medial superior temporal area in their responses to optic flow patterns. Journal of Neurophysiology 76, 4056-4068.

SchaLL, J.D. (2002). The neural selection and control of saccades by the frontal eye field. Philosophical Transactions of the Royal Society B (London) 357(1424), 1073-1082.

Schall, J.D., Morel, A., King, D.J. \& Bullier, J. (1995). Topography of visual cortex connections with frontal eye field in macaque: Convergence and segregation of processing streams. Journal of Neuroscience 15(6), 4464-4487.

Schmolesky, M.T., Wang, Y., Hanes, D.P., Thompson, K.G., Leutgeb, S., Schall, J.D. \& Leventhal, A.G. (1998). Signal timing across the macaque visual system. Journal of Neurophysiology 79(6), 3272-3278.

Segraves, M.A., Goldberg, M.E., Deng, S.Y., Bruce, C.J., UngerLEIDER, L.G. \& Mishrin, M. (1987). The role of striate cortex in the guidance of eye movements in the monkey. Journal of Neuroscience $\mathbf{7}$, 3040-3058.

SHERK, H. \& Fowler, G.A. (2001). Neural analysis of visual information during locomotion. Progress in Brain Research 134, 247-264.

Siegel, R.M., ReAD, H.L. (1997). Analysis of optic flow in the monkey parietal area 7a. Cerebral Cortex 7(4), 327-346.

Stanton, G.B., Bruce, C.J. \& Goldberg, M.E. (1995). Topography of projections to posterior cortical areas from the macaque frontal eye fields. Journal of Comparative Neurology 353(2), 291-305.

Steinmetz, M.A., Motter, B.C., Duffy, C.J. \& Mountcastle, V.B. (1987). Functional properties of parietal visual neurons: Radial organization of directionalities within the visual field. Journal of Neuroscience 7(1), 177-191.

Vidyasagar, T.R. \& HenRy, G.H. (1990). Relationship between preferred orientation and ordinal position in neurons of cat striate cortex. Visual Neuroscience 5, 565-569.

Warren, W.H., KaY, B.A., Duchon, A.P., Zosh, W. \& Sahuc, S. (2001). Optic flow is used to control human walking. Nature Neuroscience 4, 213-216.

WiLKIE, R.M. \& WANN, J.P. (2003). Eye-movements aid the control of locomotion. Journal of Vision 3(11), 677-684.

Wunderlich, G., Marshall, J.C., Amunts, K., Weiss, P.H., Mohlberg, H., Zafiris, O., Zilles, K. \& FinK, G.R. (2002). The importance of seeing it coming: A functional magnetic resonance imaging study of motion-in-depth towards the human observer. Neuroscience 112(3), $535-540$.

ZAR, Z.H. (1999). Biostatistical Analysis, fourth edition. Upper Saddle River, New Jersey: Prentice-Hall Inc. 